\title{
La Arqueología y la Antropología cultural: entrelazamientos pretéritos y presentes*
}

\author{
Archaeology and Cultural Anthropology: past and present entanglements
}

\author{
Araceli González Vázquez ${ }^{\mathrm{a}}$
}

\author{
A Alberto Gómez Castanedo y a Lydia Zapata \\ Peña. In memoriam.
}

\section{RESUMEN}

Este artículo explora los entrelazamientos pretéritos y presentes de la Arqueología y la Antropología cultural. Se centra en cuestiones de naturaleza teórica, epistemológica y metodológica. En particular, examina debates recientes ligados al llamado giro ontológico de las Ciencias Sociales y las Humanidades.

\begin{abstract}
This paper explores past and present entanglements of Archaeology and Cultural Anthropology. It focuses on issues of theoretical, epistemological and methodological nature. In particular, it examines recent debates linked to the so-called ontological turn of the Social Sciences and Humanities.
\end{abstract}

Palabras clave: Arqueología; Antropología; Interdisciplinariedad; Giro ontológico.

Key words: Archaeology; Anthropology; Interdisciplinarity; Ontological turn.

\section{INTRODUCCIÓN}

La Arqueología y la Antropología son en la actualidad dos áreas de conocimiento muy próximas y notablemente plurales, por un lado, por las circunstancias de su desarrollo teórico y metodológico en el tiempo (su historia disciplinar), y por otro lado, por la diversidad interna que presentan, al encontrarse en un momento en el que proliferan las "subdisciplinas" y las "líneas de investigación"; en el que ambas parecen oscilar entre su convergencia y su divergencia; y en el que las dos parecen caminar hacia una hiperespecialización, a la vez que hacia una considerable fragmentación.

Entre las acciones que posibilitan una cierta convergencia de ambas áreas de conocimiento se encuentran algunas tendencias generales, si bien no generalizadas, como el énfasis actual en la interdisciplinariedad, en la multidisciplinariedad, en la cross-disciplinariedad ${ }^{1}$ y en la transdisciplinariedad; el interés creciente de los arqueólogos por la teoría antropológica y por los trabajos de los antropólogos; la importancia actual de los estudios sobre la materialidad en el ámbito de la Antropología; pero también la relevancia que han ido adquiriendo subdisciplinas como la Etnoar-

\footnotetext{
* Trabajo financiado por un contrato Juan de la Cierva-Incorporación del Ministerio de Economía, Industria y Competitividad del Gobierno de España, y co-financiado por el Anneliese Maier Research Award de la Alexander Von Humboldt Stiftung/Foundation recibido (A. G. V. julio-marzo 2017) por Maribel Fierro (ILC-CCHS, CSIC) para el proyecto titulado "Practising Knowledge in Islamic societies and their neighbours".

${ }^{a}$ Dpto. de Arqueología y Antropología. Institución Milà i Fontanals (IMF), Consejo Superior de Investigaciones Científicas (CSIC). Carrer de les Egipcíaques 15.08001 Barcelona. Correo e.: araceli.gonzalez@imf.csic.es http://orcid.org/0000-0003-4241-9347.

Recibido 7-II-2017; aceptado 16-5-2017.

${ }^{1}$ Traduzco así, sólo parcialmente, el término inglés cross-disciplinarity, que resulta útil en la medida en que permite aludir a la interacción de dos o más disciplinas, haciendo referencia expresa a los cruzamientos entre ellas.
} 
queología, que grosso modo, sitúa a los arqueólogos en las sociedades del presente, o la Antropología histórica y la Etnohistoria, que sitúan a los etnógrafos, etnólogos y antropólogos en las sociedades del pasado.

¿En qué aspectos resulta evidente un entrelazamiento pretérito y presente de la Arqueología y la Antropología cultural? ¿Cuál es el presente de los espacios teórico-epistemológicos en que ambas disciplinas convergen? ¿En qué estado se hallan las reflexiones de corte teórico (y metodológico) y las pulsiones críticas?

\section{2. ¿ENTRE LA HISTORIA Y LA ANTROPOLOGÍA?}

De la expansión y consolidación histórica de ambas áreas de conocimiento, Arqueología y Antropología, emerge una diferencia básica en el modo en que se concibe la relación entre ellas, que es distinta en las tres tradiciones de investigación que mayor influencia han tenido y tienen sobre la investigación que se desarrolla en España actualmente. Tal y como es bien sabido, en la tradición francesa, y en la de muchos otros países de la Europa continental (entre ellos, también España), la Antropología -tradicionalmente denominada "Etnología"- no se formalizó en conexión con el imperialismo y con el colonialismo, como en el Reino Unido, sino con los movimientos nacionalistas de la segunda mitad del siglo XIX, como discurso y práctica sobre los orígenes, y sobre la identidad cultural de los pueblos y de las naciones $^{2}$. La institucionalización de la Ciencia generó dos disciplinas de conocimiento separadas. Tanto es así que los arqueólogos de la Europa continental se forman primordialmente como prehistoriadores o historiadores, y la Arqueología es una disciplina histórica. En Estados Unidos y Canadá, los arqueólogos se forman como antropólogos, ya que la Arqueología se desarrolló en conexión con la Antropología, en particular, como estudio sobre los pueblos indígenas del continente americano, y sobre las intensas transformaciones culturales que experimentaban en la época. Mientras que la

\footnotetext{
2 En relación con la emergencia de la Antropología, y el papel del imperialismo y el colonialismo europeos, puede consultarse: Lévi-Strauss (1960) y Bonte (1974).
}

tradición norteamericana y canadiense distingue cuatro áreas al interior de la Antropología: Arqueología, Antropología física, Antropología cultural y Antropología lingüística, en la tradición británica se reducen a tres: Arqueología, Antropología física y Antropología social. En el caso de esta última, se prefiere además este término, "social", al término "cultural", empleado con mayor frecuencia en Estados Unidos y en Canadá (Trigger 1989; Ingold 1994; Barnard 2000; Díaz-Andreu 2007; Bahn 2014). En España se suele enfatizar una vía media, también visible en otros países, que incide en la combinación de las dos ópticas, la social y la cultural, reflejada, por ejemplo, en el hecho de que los antiguos estudios de licenciatura, hoy de grado, lleven la denominación "Antropología social y cultural" en muchas universidades.

En la tradición de investigación estadounidense y canadiense, aunque la Arqueología se haya entendido tradicionalmente como parte de la Antropología (y haya sido considerada una de sus subáreas disciplinares o subdisciplinas), la relación entre ambas no siempre ha estado ni está exenta de tensiones. Basten algunos leitmotiv relevantes $\mathrm{y}$ bien conocidos para indicar que ha existido una necesidad de reafirmar posicionamientos, a la que se ha cedido con más urgencia en unos momentos que en otros. Podemos invocar una temprana afirmación de Philip Phillips (1900-1994): "New World archaeology is anthropology or it is nothing" (Phillips 1955), que luego precisa, junto a Gordon R. Willey (1913-2002), señalando lo siguiente: "American archaeology is anthropology or it is nothing" (Phillips y Willey 1958: 2), una suerte de declaración de principios enmarcada en el procesualismo. Podemos recordar el igualmente procesual "Archaeology as Anthropology" del título del conocido artículo de Lewis R. Binford (1962); así como el "Archaeology, is archaeology, is archaeology", que resume la propuesta del procesual británico David L. Clarke (1968: 13); y también un relativamente reciente "Is Archaeology Anthropology?", la certera pregunta que da título a una reflexión de Deborah L. Nichols, Rosemary A. Joyce y Susan D. Gillespie (2003), en la obra editada por Gillespie y Nichols titulada Archaeology is Anthropology, que precisamente aborda el estudio de la evolución temporal de las tensiones expresadas, bien analizadas previamente en la monografía Anthropology and Archaeology: a changing relationship de Chris Gosden (1999). 
Es bien conocido que Lewis R. Binford (1962: 224) afirmaba muy significativamente, en el citado artículo que para muchos disocia la Arqueología de la Historia y de las Humanidades, y la vincula con la Antropología y con las Ciencias Naturales, que la Arqueología debe plantearse la posibilidad de hacer avanzar el campo general de la Antropología: Archaeology must accept a greater responsibility in the furtherance of the aims of anthropology. Por su parte, la propuesta de David L. Clarke (1968: 12) enfatizaba que la Arqueología no es Historia: Archaeological data are not historical data and consequently, archaeology is not history.

Evidentemente, ni en esta época ni posteriormente, la relación de la Arqueología con la Historia se entiende de forma anómica. Tanto es así, que en parte por el efecto de las tensiones mencionadas, encuadrables en el procesualismo, se llega a proponer concebir a la Arqueología como una disciplina de conocimiento completamente independiente, de ninguna manera supeditada a la Historia ni a la Antropología social y cultural. Esta propuesta, que no es mayoritaria, y que se afirma sin ambages, por ejemplo, en la obra de David L. Clarke, ha planteado y plantea oposiciones, particularmente entre quienes estiman que ello le restaría pulso a la Arqueología, al menguar los réditos de la interdisciplinariedad y del fértil diálogo que mantienen las Ciencias Humanas y Sociales. En cierto modo, se trata de una propuesta también derivada de la intensa aproximación metodológica de la Arqueología a las Ciencias Naturales (Química, Física, Geología, Mineralogía, Zoología, Botánica, entre otras), notable en dos momentos: el de la Nueva Arqueología de los años 1960 y 1970, en el que se enmarcan los postulados de Clarke; y el momento actual.

La plena identificación de la Arqueología con la Antropología también se ha estimado problemática, como refleja el particularismo por el que aboga Ian Hodder (1986) en Reading the past, reafirmando lo expuesto por Clarke y reeditado en la nueva edición de la obra: ... archaeology is neither history not anthropology, but just archaeology (Hodder y Hutson 2003: 243).

Los debates que evidencian las tensiones entre las disciplinas ayudan a comprender que la Arqueología tiene muy poco hoy en día de subgénero/subdisciplina de la Antropología o de la Historia, en particular si se tiene en cuenta lo que aporta a su propio avance y al avance de las otras dos disciplinas mencionadas, y la existencia de una agenda propia, no supeditada a los debates externos, sino desarrollada a través de interesantes formas de entrelazamiento con ellos (véase una reciente e interesante reflexión sobre Arqueología e Historia en Herschend 2015).

Es cierto que aún muchos investigadores piensan a los arqueólogos como antropólogos culturales o historiadores centrados en el pasado, a la vez que piensan a los etnógrafos y antropólogos sociales o culturales como investigadores centrados únicamente en el presente. A este respecto hay que señalar que la cuestión de la temporalidad (pasado vs. presente) va dejando de ser, progresivamente, la cuestión esencial para señalar los centros de interés de las dos disciplinas de conocimiento y de los investigadores (véase, sobre la relación entre la Antropología y la Historia, el libro de Mateo Dieste y Coello de la Rosa 2016). Tradicionalmente, la Arqueología ha sido considerada una disciplina centrada en el estudio de la humanidad en el pasado, pero cada vez con mayor insistencia, los arqueólogos se formulan preguntas sobre el presente y el futuro de la humanidad, y tratan de ofrecer respuestas a cuestiones que son relevantes para las sociedades actuales y para su futuro en la Tierra. También, cada vez con mayor frecuencia, los arqueólogos se interesan por las sociedades del presente. Antes que el estudio del pasado, la Arqueología es el estudio de la materialidad, de los restos generados por los seres humanos en los procesos de producción de la vida material y social, particularmente a partir de sus interacciones intraespecíficas e interespecíficas y extraespecíficas: consigo mismos, con otros seres y con el entorno, a lo largo de la Historia (y en la Prehistoria, como tiempo histórico) y en el presente. Las dos áreas de conocimiento, la Arqueología y la Antropología cultural, estudian el pasado y el presente de la humanidad (la Arqueología quizá con mayor énfasis en el pasado y en la materialidad, y la Antropología quizá con mayor énfasis en el presente y en la relacionalidad), pero fundamentalmente abordan hoy en día lo que supone ser o llegar a ser humano, y las relaciones que conciernen a los humanos, a los no-humanos (animales, vegetales, y muchos otros) y al entorno físico en el que se 
desenvuelven todos ellos ${ }^{3}$. Ambas áreas de conocimiento fijan su atención, de forma holística y hermenéutica, en la diversidad de la experiencia humana, aportando conocimientos críticos sobre ella. Así las cosas, no cabe limitar la Arqueología a la producción de lecturas sobre el pasado, sino a la generación de discursos y prácticas aptas para abordar el pasado y el presente.

La cuestión de la alteridad es de una enorme relevancia y aparece en el centro de las reflexiones sobre las dos áreas de conocimiento, particularmente de las vinculadas con la forma en que ambas permiten avanzar en la comprensión de las formas de ser, ontológicas o existenciales, y de las formas de conocer, epistemológicas. O, dicho de otro modo, profundizar en lógicas ónticas y epistémicas.

Tanto la Arqueología como la Antropología cultural se encuentran actualmente en un momento de (re)orientación teórica y metodológica. En lo básico, las dos disciplinas han generado en su seno múltiples críticas del eurocentrismo (o de la hegemonía occidental y el colonialismo), del androcentrismo y, en especial en el último lustro, del antropocentrismo inherente a ellas, con posiciones a favor de un descentramiento del anthropos (y, especificamente, de un intenso cuestionamiento de la concepción androcéntrica del anthropos) y de una suerte de "repoblamiento" del foco de interés. Este "repoblamiento" hace referencia a una mayor toma de conciencia sobre las agencias de los seres no-humanos y de los materiales, pero también $-\mathrm{y}$ con singular énfasis- habla a favor de una presentación más compleja de las realidades humanas y no-humanas, incluyendo en las narrativas sobre el pasado o sobre lo material a individuos y colectivos antes ignorados. Un ejemplo clave es el de las mujeres y la Antropología/Arqueología del Género y la Antropología/Arqueología Feminista; otro elocuente es el de la Antropología/Arqueología del cuerpo, que sitúa su centro de interés en la fisicalidad (fundamentalmente, pero no sólo, en la humana) y que analiza la inscripción corporal de los procesos sociales y culturales; y, finalmente, otro de certera pujanza es el ejemplo de los animales y la animalidad, una cuestión que emerge

\footnotetext{
${ }^{3}$ Muy significativamente, en la ruptura con las constricciones temporales llegan a definirse áreas de investigación, al interior de la Arqueología, como la "arqueología del pasado contemporáneo" (Archaeology of contemporary past) o la "arqueología del pasado reciente" (Archaeology of the recent past).
}

con fuerza y es considerada desde puntos de vista plurales, tanto por arqueólogos como por antropólogos e historiadores. En parte, esto es así por una implosión de estudios realizados al hilo de aquello que se propone como "giro animal" (ing. animal turn, fr. tournant animaliste), pero no únicamente.

La época actual, considerada postpositivista y posthumanista, es una época de establecimiento de nuevas sinergias entre las dos áreas de conocimiento, Arqueología y Antropología cultural. A su vez, es una época en que la emergencia del capitalismo neoliberal, especialmente a partir de los años 1980, ha tenido un impacto muy profundo en las Ciencias Humanas y Sociales. En lo que son, pero también en lo que pueden devenir en un futuro que parece sombrío. En las perspectivas teóricas desde las que se trabaja, pero también en la propia práctica laboral. Es particularmente interesante, en el contexto de intensa crisis actual, el hecho de que el neoliberalismo se constituya como objeto de investigación de las dos disciplinas, y que a la vez sea el marco en el que tiene lugar la propia producción y la transmisión del conocimiento arqueológico y antropológico (Comaroff y Comaroff 2001; Bauman 2007; Hamilakis y Duke 2007).

Por otro lado, en ambas disciplinas, Arqueología y Antropología cultural, existen proyectos epistemológicos y políticos que cuestionan intensamente las propuestas hegemónicas y dominantes. Ejemplos significativos son los de las Antropologías "del sur" y las Antropologías "segundas" (Krotz 1997, 2015); las Antropologías "otras" y de "otro modo" (del ing. otherwise) (Restrepo y Escobar 2005); y las Antropologías "del mundo" (Ribeiro y Escobar 2008), entre algunas otras, como las Antropologías y Arqueologías "periféricas" y "decoloniales".

\section{DE “GIROS" E INFLUENCIAS MUTUAS}

La Antropología es una de las ciencias sociales que mayor influencia ejercen sobre las disciplinas de conocimiento próximas a ella, particularmente sobre la Arqueología. A su vez, es una de las ciencias sociales más conectadas con la Filosofía, un área disciplinar que ha nutrido hasta la fecha, con diferente insistencia, buena parte de sus problemáticas y de sus lógicas. 
Desde la segunda mitad del siglo XIX, y a lo largo del siglo XX y de lo que va del siglo XXI, desde un punto de vista teórico, pero también con una clara influencia sobre aspectos de la praxis, sobresalen los aportes del evolucionismo (Morgan, Tylor, Frazer), del difusionismo (Kroeber, Wissler, Smith, Perry, Rivers) y particularismo histórico (Boas), del funcionalismo (Malinowski, EvansPritchard), del estructuro-funcionalismo (Radcliffe-Brown, Leach, Firth, Fortes), del culturalismo (Mead, Benedict), del marxismo y del neomarxismo (Meillassoux, Godelier, Terray, Rey, Bonte), de la antropología dinámica (Bastide, Balandier), del materialismo cultural (Harris, Sahlins) y la ecología cultural (Steward, White), del estructuralismo (Lévi-Strauss, Bourdieu, Dumont, Needham, De Heusch, Héritier), del interpretativismo-simbolismo (Schneider, Geertz, Douglas, Turner), del feminismo (Irigaray, Butler, Braidotti, Haraway, Kristeva, Strathern, Moore), del postcolonialismo, del postestructuralismo y del posthumanismo.

En la última década se ha ampliado notablemente la reflexión teórica y epistemológica generada en conexión con aquello que se ha dado en denominar el "giro ontológico" (ing. ontological turn, fr. tournant ontologique) de la Antropología contemporánea $\mathrm{y}$, en general, de las Ciencias Sociales y de las Humanidades (Viveiros de Castro 2003; Henare et al. 2007; Alberti et al. 2011; véanse, las múltiples reflexiones sobre el "giro", entre otros, en Course 2010; Laidlaw 2012; Pedersen 2012; Ramos 2012; Laidlaw y Heywood 2013; Woolgar y Lezaun 2013, 2015; Bessire y Bond 2014; Vigh y Sausdal 2014; Kohn 2015; una amplia reflexión en castellano sobre el "giro ontológico" en González Abrisketa y Carro-Ripalda 2016, y una revisión en profundidad en inglés en la reciente monografía de Holbraad y Pedersen 2017). Tal y como trataré de explicar, la Arqueología, dada su íntima relación con la Antropología cultural, no permanece al margen de tales reflexiones, ni de los debates de los que emergen actualmente nuevas maneras de pensar y de trabajar, en particular de superar la oposición dicotómica entre naturaleza y cultura y cualesquiera otros dualismos desde los que se trabajara en el pasado (cuerpo/mente, sujeto/objeto, entre muchos otros). En buena medida, este llamado giro ontológico engloba reacciones acusadamente heterogéneas frente al "giro lingüístico", el "giro reflexivo o interpretativo" y la Postmodernidad, y aunque en cierta medida, y en algunos casos, se autopostule como ruptura, viene posibilitado (y generado) por avances previos, anticipados, por ejemplo, en las obras de Roy Wagner (1978, 1981), Donna J. Haraway $(1991,2003,2007)$ y Marilyn Strathern (1988, 1991, 1995), que cuajan, con diferente entidad, y que dialogan, en diferente medida, con los trabajos de investigadores como Bruno Latour (Latour y Woolgar 1979; Latour 1987, 1988, 1991, 1996, 1999a, 1999b, 2005, 2012, 2013a, 2013b), Philippe Descola (1992, 1996, 2005; Descola y Pálsson 1996), Tim Ingold (2000, 2007, 2011, 2012, 2013; Ingold y Pálsson 2013), Eduardo Viveiros de Castro $(1998,2003,2009,2011)$ y Martin Holbraad (2012), entre otros y entre otras de sus obras. También por la influencia que ejercen las obras filosóficas de Martin Heidegger, Gilles Deleuze, Félix Guattari y Alfred North Whitehead.

En cualquier caso, el grado de adhesión de estos y otros investigadores a las propuestas nodales o nucleares del proyecto es muy variable. En torno a este proyecto, en caso de que fuera unívoco y podamos hablar de él en singular, cosa harto dudosa, surgen algunos otros proyectos concretos interesantes, como la "etnografía multiespecífica" de S. Eben Kirksey y Stefan Helmreich (2010), y la "antropología de la vida" de Eduardo Kohn (2013). Todos ellos contribuyen a consolidar la crítica postestructuralista, postconstructivista y posthumanista de la investigación antropológica. En primera instancia, ponen de relieve que la historia de la disciplina antropológica se ha constituido sobre una oposición, la distinción naturaleza/cultura, que estructura el mundo de la "modernidad", en particular el de la "modernidad occidental", y que constriñe decididamente la forma en que los antropólogos comprendemos la diversidad de la experiencia humana. Una de las principales contribuciones del giro ontológico se halla en la forma en que intensifica o radicaliza ciertos potenciales existentes previamente, pero que en buena parte permanecían dormidos en el proyecto antropológico. También, como veremos, en el arqueológico.

En buena medida, el así llamado giro ontológico ha contribuido a "repoblar las Ciencias Sociales" (Thiéry y Houdart 2011) o a "(re) animar el mundo" (Blaser 2014), intensificando la atención en los seres no-humanos, y situando en su seno otros movimientos intelectuales desarrollados en el marco de las Ciencias Sociales y las Humanidades, como el llamado giro 
social (ing. social turn), el epistemológico (ing. epistemological turn), el material (ing. material turn), el animalista (ing. animal turn, fr. tournant animaliste), y el giro (hacia lo) no-humano (ing. non-human turn). Todos ellos forman parte de lo que el arqueólogo Christopher Witmore (2015: 38) considera, no sin vis crítica, una "letanía" de giros. Una crítica muy explícita se manifiesta en los títulos de los trabajos de otros arqueólogos y antropólogos, y por supuesto, en las reflexiones que aportan, como en "One more turn and you're there" de James Laidlaw y Paolo Heywood (2013) y en "Ontology, 'hauntology', and the 'turn' that keeps anthropology turning" de Vassos Argyrou (2017). En el ámbito de los estudios feministas también han emergido voces críticas con la "inflación" de "giros", que proponen analizar el tipo de labor discursiva que está detrás del uso de la metáfora del "giro", y que proponen evaluar las consecuencias epistemológicas y políticas de este uso. A este respecto el editorial de Kathy Davis (2015: 125-128) "The politics of the "turn"” en la revista European Journal of Women's Studies señala en qué medida es pernicioso que los "giros" contribuyan a clasificar la producción de conocimiento en un "antes" y un "después". Igualmente, se han formulado críticas hacia la metáfora del "giro" desde los estudios de ciencia y tecnología (STS, ing. Science and Technology Studies), por ejemplo, las de Bistra Vasileva (2015) en su artículo "Stuck with/in a "turn": Can we metaphorize better in Science and Technology Studies?"

La Arqueología de las últimas décadas también ha registrado un fuerte impacto de los que el arqueólogo Christopher Witmore (2014) ha denominado "nuevos materialismos", no marxistas o neomarxistas, sino enraizados-en y enlazados-con el "giro ontológico" y con otros nódulos teóricoepistemológicos. En este marco, algunos arqueólogos proponen que la Arqueología no es el estudio del pasado humano a través de los restos materiales, sino una verdadera "disciplina de las cosas" (Olsen et al. 2012), una suerte de ecología material de las prácticas humanas y no-humanas. Aquí resulta de gran influencia la publicación del volumen Thinking through things (Henare et al. 2007), pero también la llamada "teoría del ensamblaje" (assemblage theory), vía Gilles Deleuze y Félix Guattari (1980) y Manuel DeLanda (2006) (Fowler 2013; Hamilakis 2013; Lucas 2012; Jones y Alberti 2013). Una buena parte de los nuevos ma- terialismos están vinculados con la práctica de una "arqueología simétrica", definida bajo la influencia de los planteamientos del sociólogo francés Bruno Latour (Shanks 2007; Witmore 2007, Olsen 2012b; Olsen y Witmore 2015; Preucel 2016).

Una serie de conceptos se sitúan como ejes de trabajo. O la "cosa" o el "ensamblaje" devienen, en estas orientaciones teóricas, conceptos centrales en la reflexión de los arqueólogos. No menos importante resulta el concepto de "agencia" (Gell 1998). A través de la práctica arqueológica, se procuraría explorar y reconocer el grado de agencia de las materias y los materiales, de los objetos/cosas/artefactos, y de los espacios/lugares/paisajes/entornos. Igualmente se constata una influencia reciente del "nuevo realismo ontológico" (Gabriel 2015; Alberti 2016). Los nuevos entrelazamientos y entrecruzamientos son explícitos en numerosos trabajos publicados en la última década en el ámbito de la Arqueología, en trabajos que, desde distintos planteamientos, sitúan en los "objetos", en las "cosas", o en los "ensamblajes", su foco de interés (Webmoor y Witmore 2008; Olsen 2003, 2010; Alberti 2014, Alberti y Marshall 2009, Marshall y Alberti 2014; Fowler 2013, Fowler y Harris 2015). En muchos estudios que emplean el concepto de "agencia" se deja sentir con fuerza la influencia del "realismo agencial" que plantea Karen Barad (2003, 2007).

La influencia de los STS (ing. Science and Technology Studies) y de la OOO (ing. Object Oriented Ontology) es notable. Hay, no obstante, una diferencia fundamental entre esta última propuesta y las de arqueólogos como Bjørnar Olsen y Christopher Witmore, más centradas en la idea de materialidad, y no en la cuestión de la forma, que es una cuestión que trata la $O O O$ (Bryant 2011, Bryant et al. 2011; Edgeworth 2016; Harman 2005, 2009), en parte por influencia del pensamiento del filósofo francés Gilbert Simondon (1964), autor de L'individu et sa génèse physico-biologique. En los últimos dos años, Ian Hodder, icono de la Arqueología postprocesual, desarrolla además la idea del entanglement (entrelazamiento), principalmente frente a los postulados de la teoría del actor-red (ing. actor-network theory, ANT) de Bruno Latour, que permea los trabajos de Witmore (2007) y otros. En su libro Entangled: an Archaeology of the relationships between humans and things, Hodder (2012) asume las influencias de la antropóloga Marilyn Strathern y su énfasis en la relacionalidad

Trab. Prehist., 74, N. ${ }^{\circ}$ 2, julio-diciembre 2017, pp. 211-224, ISSN: 0082-5638

doi: $10.3989 /$ tp.2017.12191 
del sociólogo Bruno Latour y del filósofo Pierre Lemonnier. La obra es un ejemplo de la ausencia de un paradigma sólido que sustituya a los previos, al encontrarnos en un momento en el que se formulan propuestas que son suplementarias. Hodder (2016) también profundiza en su conceptualización del "entrelazamiento" en su mas reciente Studies in human-thing entanglement, que refleja perfectamente la integración de enfoques de la Arqueología y la Antropología cultural.

Las posiciones críticas también son interesantes. En una reflexión reciente, Severin Fowles (2016) afirma que la expansión de la "teoría de la cosa" (ing. thing theory, Brown 2001) no se entiende sin la crítica postcolonial, y que a través de la primera los arqueólogos han explotado las ventajas de "tratar a los objetos no-humanos como sujetos cuasi-humanos": según él, al modificar el foco del análisis, de la gente a las cosas, se conserva la autoridad representacional de los académicos occidentales en un momento en que el cuestionamiento de esta autoridad es particularmente intenso. También crítico con estos planteamientos, Artur Ribeiro (2016) habla en fechas recientes de "tiranía de la cosa".

Aunque algunas propuestas del llamado giro ontológico se formulen, explícitamente, como un "retorno a las cosas", y aunque el diálogo entre arqueólogos y antropólogos sea fértil en su seno, la diversidad impera y no intensifica de modo claro el acercamiento entre ambas áreas de conocimiento. De hecho, desde "el giro" en Arqueología, Olsen (2012a) proclama un "retorno a la arqueología", y Witmore (2007) subraya que la fuerza de la disciplina descansa en lo que los propios arqueólogos hacen. En los estudios sobre la cultura material o la materialidad, se habla de un verdadero "retorno hacia las evidencias arqueológicas", de un regreso del empiricismo, o de un neoempiricismo (Hillerdal y Siapkas 2015), pero también de un regreso del realismo, o neorrealismo. En ámbitos antropológicos, las propuestas neoempiricistas y neorrealistas son evidentes en corrientes como, por ejemplo, el realismo especulativo (ing. speculative realism) (Bryant et al. 2011) o el nuevo realismo ontológico (ing. new ontological realism).

Al interior de los "nuevos materialismos" se define también la existencia de un "giro feminista", patente sobre todo en la forma en que se incorpora en Arqueología el "materialismo feminista" o los "feminismos material(es)/(istas)" de las propuestas de Donna Haraway, Rosi Braidotti, Elisabeth Grosz, Karen Barad y Claire Colebrook, entre otras investigadoras.

La llamada Nueva Arqueología de los años 1960 y 1970 (Binford 1962; Clarke 1972; Binford y Binford 1968) (luego denominada "Arqueología procesual" o "Procesualismo"), surge como crítica del paradigma histórico-cultural y de su positivismo, que era la ortodoxia y la ortopraxis del momento. Las reacciones frente a ella, que surgen con mayor brío a mediados de los 80 , forman la trama y urdimbre de lo que se ha dado en llamar Arqueología postprocesual (Hodder 1982a; Shanks 1992 y Shanks y Tilley 1987a, 1987). En este cul-de-sac postprocesual se suelen inscribir los aportes estructuralistas y postestructuralistas (incluido el postestructuralismo marxista), marxistas y neomarxistas, feministas, indigenistas y postcoloniales, marcados por el constructivismo, por el relativismo científico, y por el individualismo filosófico, así como por el énfasis en la subjetividad y en la interpretación. En las tres últimas décadas del siglo XX, esta orientación se desarrolla dentro de lo que se denomina convencionalmente el "giro postmoderno". En Arqueología, la mayor parte de las corrientes a las que engloba se constituyen como críticas de la ortodoxia procesual de la Arqueología norteamericana y británica. Entre otros, destacan los aportes de Ian Hodder (1982a, 1982b, 1982c, 2012, 2016, entre otros trabajos), de Michael Shanks (1992), de Christopher Tilley (1994, 1999), de ambos de forma conjunta (Shanks y Tilley 1987a, 1987b), de Robert W. Preucel (2006) y de Daniel Miller (1987, 1998, 2005), de una nómina de arqueólogos estructuralistas y neomarxistas, como Bruce Trigger (1993, 1995), Matthew Spriggs (1984), Antonio Gilman (1984), Robert Paynter (1988), y Randall H. McGuire (1992, 2009; McGuire y Paynter 1991), y feministas, como Margaret Conkey (2003; Conkey y Spector 1984), Joan M. Gero (Gero et al. 1991), Janet Spector (1991) y Alison Wiley (1991). La Arqueología postprocesual ha contribuido de forma clara a que la Arqueología participe de las posiciones teóricas compartidas por las Humanidades y las Ciencias Sociales, posicionándose como hermenéutica y subrayando la pertinencia de un conocimiento fenomenológico del pasado.

En Arqueología, resulta bastante evidente que se está produciendo una intensa aproximación metodológica de la disciplina a las Ciencias Naturales (Química, Física, Geología, Mineralogía, Zoología, 
Botánica, entre otras), notable ya en un momento anterior, el de la Nueva Arqueología de los 1960 y 1970, pero especialmente en el actual. A partir de las innovaciones en las técnicas y las tecnologías científicas de las últimas décadas, incorporadas de forma creciente, las prácticas arqueológicas (también las etnográficas) y los métodos de investigación se han diversificado y han permitido notables avances en el conocimiento factual, pero también en el conocimiento problemático. En la Arqueología se han desarrollado enormemente algunas subdisciplinas, como la Bioarqueología, y líneas de investigación que incorporan de forma decidida los métodos de trabajo de las Ciencias Naturales. En el presente, los materiales arqueológicos se estudian a través de múltiples técnicas de análisis, con un elevado grado de sofisticación. A su vez, proliferan las reflexiones sobre el estado de las disciplinas y sobre las teorías, como ejemplifican, en Arqueología, los trabajos recientes de Alfredo González-Ruibal (2008, 2013, 2014), Benjamin Alberti y otros (2013), Ian Hodder (2012, 2016), Bjørnar Olsen (2010, 2012a) y Christopher Witmore (2014), entre varios otros. Algunos estudiosos consideran que "se ha detenido" el desarrollo de la teoría (Shennan 2004), que la teoría antropológica podría haber "muerto" (véase el título de Bintliff y Pearce 2011), que nos encontramos en una época "postparadigmática" (Knauft 2006) o "post-teorética" (el término es de Siapkas y Hillerdal 2015, de su visión crítica de estos argumentos sobre el estancamiento de lo teorético) o en una época en la que no existe un paradigma explícito (Criado-Boado 2016).

Visto desde el presente, parece claro que el peso que ha adquirido el postprocesualismo dentro de la Arqueología ha servido para generar una suerte de versión mainstream del hacer arqueológico y de las propuestas heurísticas. A este acontecer, Knauft (2006) le llama giro hacia "articulaciones postparadigmáticas", aunque también hay publicaciones recientes, como el reader editado por Kristian Kristiansen, Ladislav Šmejda y Jan Turek (2015), que debaten la pérdida y la recuperación del paradigma con menor escepticismo, anunciando un reencuentro: "Paradigm found". Muy probablemente una recuperación de paradigma que se percibe ligada a la emergencia del "giro ontológico". En cualquier caso, tal y como ha hecho recientemente Artur Ribeiro, cabe preguntarse si la Arqueología necesita de un constante cambio de paradigma, si en realidad, en fechas recientes, ha tenido lugar cambio de paradigma alguno (Ribeiro 2016: 149), y en qué medida se está produciendo una suerte de fetichización de lo nuevo y de lo innovador (Ribeiro 2016: 147).

Lo reflexivo, lo deconstructivo y lo crítico se ha ido instalando en la academia (no en todas las academias por igual, y de ello da especial cuenta el status quo en España) y ha devenido "normal". Incluso se ha producido un cierto declive del debate teórico explícito, que algunas voces intentan paliar. En cierto modo, lo que se establece en los noventa es una suerte de consenso (un "pluralismo consensual", como lo ha definido Lucas 2016, o un "nuevo pragmatismo", como señalan Preucel y Mrozowski 2010), a partir de la hibridación de los aportes procesuales y postprocesuales más estimados, y tal y como señalaron Olsen et al. (2012), una cierta "trivialización de la teoría", que habría perdido filo crítico o, en opinión de Kristiansen (2004), una "brecha teórica" (ing. theoretical gap), resultante de haber aceptado el desarrollo de enfoques epistemológicamente incompatibles, que habrían hecho necesario revivir un debate teórico durmiente. Esta brecha es, por ejemplo, la que separa una arqueología evolucionista y una arqueología interpretativa, entre las que algunos arqueólogos, como Cochrane y Gardner (2011), proponen un diálogo. De acuerdo con el historiador de la arqueología Bruce G. Trigger (1989), la disciplina viene marcada además por una dicotomía entre el idealismo y el materialismo. Kristiansen (2004), por el contrario, entiende que la dicotomía contrapone perspectivas orientadas al sujeto y a la agencia, frente a perspectivas orientadas a la ciencia.

En un artículo en el que se pregunta por el futuro teórico de la Arqueología, Julian Thomas (2015: 1287) estima que en las últimas décadas se ha producido una aceleración del desarrollo de la disciplina, resultado en su opinión de la "asimilación del aparato conceptual de las ciencias naturales y humanas", y que, a la vez, la Arquelogía se encuentra plenamente implicada en los debates filosóficos de las Humanidades. En Antropología existe una clara intensificación de la reflexión teórica, de notable impacto si se piensa en la difusión que alcanza la revista $\mathrm{Cul}$ tural Anthropology, que ha devenido de acceso abierto (ing. open access), y que ha publicado en fechas recientes números de corte teórico y crítico de notable impacto, y la revista HAU, Journal

Trab. Prehist., 74, N. ${ }^{\circ}$ 2, julio-diciembre 2017, pp. 211-224, ISSN: 0082-5638

doi: $10.3989 /$ tp.2017.12191 
of Ethnographic Theory, que supone un potente revulsivo. Es una tendencia que se puede considerar plenamente abierta en 2008 , en los debates sobre la disciplina que se producen en el seno del Group for Debates in Anthropological Theory (GDAT) de Manchester, en particular alrededor de las nociones de ontología y cultura ("Ontology is just another word for culture"). La Antropología cultural, que ha sido concebida tradicionalmente como una ciencia social sincrónica, centrada en grupos humanos concretos, y fundamentada en el trabajo de campo etnográfico, experimenta en las últimas décadas una notable reconfiguración. En un momento profundamente autorreflexivo, el del postmodernismo, los antropólogos se cuestionaron los modos de representación de la alteridad tradicionales. Varias monografías publicadas desde el ámbito de la Arqueología inciden en la idea de que se han superado marcos teóricos previos, como Archaeology beyond Postmodernity de Andrew M. Martin (2013), o Archaeology after interpretation de Benjamin Alberti y otros (2013).

Una de las principales preocupaciones del momento es la de escribir etnografías genuinas, lo que genera una profunda renovación de la escritura y de la práctica etnográfica, que en ocasiones recibe el nombre de "nueva etnografía" (ing. new ethnography). A la par que una renovación en la escritura, se le otorga un mayor énfasis a la pertinencia de la analogía etnográfica. Esto es especialmente visible en algunos debates concretos, como el que emerge en torno a la noción y cuestión del "animismo" (denominado, en algunas instancias, "nuevo animismo", ing. new animism) (Bird-David 1999, Harvey 2005, Willerslev 2007, 2012). Del impacto de este debate en Arqueología da cuenta el volumen editado por Benjamin Alberti y Tamara L. Bray (2009) "Animating archaeology: of subjects, objects and alternative ontologies".

En la actualidad también parece existir un doble énfasis, en la teorización a partir de la etnografía (ing. ethnographic theory) y/o en el abandono de la etnografía (Ingold 2014). Igualmente proliferan las antropologías y las arqueologías de la diferencia, comprometidas con una mejor comprensión de la multiplicidad de los mundos humanos y no-humanos, algunas con una insistencia explícita en la noción de worldling. Ello haría vigente, para el sentir actual, el dictum de Ruth Benedict: The purpose of Anthropology is to make the world safe for human differences. Es notoria también la influencia del "perspectivismo", tal y como lo plantea Viveiros de Castro (1998) a partir de sus estudios en la Amazonía.

\section{CONCLUSIONES}

A partir de las innovaciones en las técnicas y las tecnologías científicas de las últimas décadas, incorporadas de forma creciente, las prácticas arqueológicas y también las etnográficas y antropológicas, los métodos de investigación particularmente, se han diversificado y han permitido notables avances en el conocimiento factual. En la Arqueología se han desarrollado subdisciplinas, como la Bioarqueología, por poner un ejemplo, y líneas de investigación que incorporan de forma decidida los métodos de trabajo de las Ciencias Naturales (físicas, biológicas, ambientales...). En Antropología cultural y social, algunas áreas subdisciplinares o subáreas, han alcanzado desarrollos muy notables, como la antropología médica o de la salud y la antropología visual. Hay un resurgimiento de nuevos-viejos temas, como la hospitalidad (Deleuze), la magia (Malinowski, Mauss, Evans-Pritchard, Taussig, Kapferer, Luhrmann), y un largo etc.

En lo que se refiere a la evolución futura de las dos áreas de conocimiento, Arqueología y Antropología cultural, es importante considerar el actual contexto económico, social y político, de inicio en 2008 , de crisis y de elevada incertidumbre sobre el progreso, sobre la estabilidad de la investigación, y sobre la estabilización laboral y profesional de los individuos, colectivos e instituciones que investigan. Tanto la Arqueología como la Antropología cultural son disciplinas de conocimiento cuyo desarrollo depende de las inversiones económicas, principalmente estatales, pero también crecientemente privadas. Junto a los avances teóricos y metodológicos, en particular por el desarrollo de nuevas técnicas de análisis y por una ampliación de los enfoques críticos y de su permeabilidad respecto de los debates teóricos de la Antropología, la Arqueología experimenta un fuerte impacto de la crisis económica: reducción de las inversiones en investigación, y por tanto de los recursos con que cuentan los investigadores y los equipos de investigación. Igualmente, en este contexto de creciente constricción política, se ejerce una influencia determinante sobre aquello que se estudia, y sobre la 
forma en que se estudia, en muchos casos condicionando las agendas investigadoras. Lejos de resultar plenamente constrictora, esta influencia está dando lugar a una contestación muy fértil, haciendo que las disciplinas centren su atención en cuestiones relevantes para las agendas políticas del presente y del futuro: la crisis ecológica, las dislocaciones rurales y urbanas, las conexiones y desconexiones globales, la desigualdad social y económica, las etnografías y antropologías de los movimientos sociales de Oriente Próximo/Oriente Medio (“¿Primaveras árabes?") y los conflictos violentos/bélicos subsiguientes; los movimientos sociales en Europa occidental (Indignados, Nuit debout...) y de los Estados Unidos (Occupy); pero también del impacto de la austeridad, de la incertidumbre, y otros. Buena parte de estos temas y otros se encuentran en la agenda de los antropólogos por la influencia que ejercen algunos filósofos postmarxistas en las Ciencias Sociales y las Humanidades, como Antonio Negri, Alain Badiou, Slavoj Žižek, Ernesto Laclau y Jacques Rancière. De particular interés son los debates en torno a lo que se ha dado en llamar el Antropoceno (Crutzen y Stoermer 2000).

En el presente de la Arqueología y la Antropología cultural puede hablarse de "arqueologías" y de "antropologías", haciendo referencia al grado en que las dos disciplinas se han convertido en campos heterogéneos que albergan perspectivas muy diversas. Una de las realidades del presente de la Arqueología es, precisamente, la amplia aceptación que genera la formulación de arqueologías "múltiples" o "plurales", que pueden interpretarse como síntoma de la diversificación, de la fragmentación y de cierto eclecticismo, pero también del extraordinario pluralismo teórico-metodológico. Otra de sus realidades es una suerte de hibridación teóricometodológica que se percibe de forma positiva. En una reflexión reciente, Felipe Criado Boado (2016) plantea que la arqueología precisamente requiere de una amalgamación de humanidades y de ciencia, de narrativa y de conocimiento científico.

En el futuro de las dos disciplinas seguirán siendo muy relevantes las innovaciones tecnológicas y técnicas, tanto para la producción del conocimiento, como para su transmisión y socialización. En el presente, y también en el futuro inmediato, la evolución de ambas se verá marcada por el impacto de las nuevas formas de producción y transmisión del conocimiento científico, con nuevas estrategias de comunicación y difusión, que pasan por una socialización del conocimiento más eficiente y por el acceso abierto y gratuito. En buena medida, la Arqueología y la Antropología cultural se desarrollan principalmente en marcos neoliberales y del capitalismo tardío. Si se produjeran tranformaciones sociopolíticas profundas, las dos disciplinas experimentarían una profunda transformación. Existe ya un proyecto descolonizador activo, que surge de las luchas contrahegemónicas de los profesionales del llamado Tercer Mundo y de los colectivos minorizados en Occidente, y de los diálogos y debates que mantienen profesionales de todo el mundo. También de proyectos teóricos explícitos, como el de Eduardo Viveiros de Castro (2009), expresado en sus Métaphysiques cannibales, de una descolonización del pensamiento antropológico.

\section{AGRADECIMIENTOS}

Este artículo ha sido realizado gracias a un contrato Juan de la Cierva-Incorporación que me permitió trabajar, bajo la supervisión de Maribel Fierro (ILC-CSIC), en el Instituto de Lenguas y Culturas del Mediterráneo y Oriente Próximo (ILC) del Centro de Ciencias Humanas y Sociales (CCHS) del CSIC, en Madrid, entre los meses de julio de 2016 y marzo de 2017. Quiero expresarle a Maribel Fierro mi sincero agradecimiento por su apoyo.

\section{BIBLIOGRAFÍA}

Alberti, B. 2014: "Archaeology, risk, and the alter-politics of materiality". Theorizing the Contemporary, Cultural Anthropology website, January 13, 2014. https://culanth.org/fieldsights/476-archaeology-risk-and-the-alter-politics-of-materiality

Alberti, B. 2016: "Archaeologies of Ontology". Annual Review of Anthropology 45: 163-179.

Alberti, B. y Bray, T. L. 2009: "Animating Archaeology. Of subjects, objects and alternative ontologies". Cambridge Archaeological Journal 19 (3): 337-441.

Alberti, B. y Marshall, Y. 2009: "Animating archaeology: local theories and conceptually open-ended methodologies". Cambridge Archaeological Journal 19 (3): 344-356.

Alberti, B.; Fowles, S.; Holbraad, M.; Marshall, Y. y Witmore, C. 2011: "Worlds otherwise: archaeology,

Trab. Prehist., 74, N. ${ }^{\circ}$ 2, julio-diciembre 2017, pp. 211-224, ISSN: 0082-5638

doi: $10.3989 /$ tp.2017.12191 
anthropology, and ontological difference". Current Anthropology 52 (6): 896-912.

Alberti, B.; Jones, M.; y Pollard, J. (eds.) 2013: Archaeology after interpretation. Returning materials to archaeological theory. Left Coast Press. Walnut Creek.

Argyrou, V. 2017: 'Ontology, 'hauntology', and the 'turn' that keeps anthropology turning". History of the Human Sciences 30(1): 50 - 65.

Bahn, P. (ed.) 2014: The History of Archaeology: an introduction. Routledge. Londres.

Barad, K. 2003: "Posthumanist performativity: how matter comes to matter". Signs: Journal of Women in Culture and Society 28 (3): 801-31.

Barad, K. 2007: Meeting the universe halfway: Quantum Physics and the entanglement of matter and meaning. Duke University Press. Durham (NC).

Barnard, A. 2000: History and theory in Anthropology. Cambridge University Press. Cambridge.

Bauman, Z. 2007: Consuming life. Polity. Cambridge.

Bessire, L. y Bond, D. 2014: "Ontological anthropology and the deferral of critique". American Ethnologist 41 (3): 440-456

Binford, L. R. 1962: "Archaeology as Anthropology". American Antiquity 28 (2): 217-225.

Binford, L. R. 1972: An Archaeological Perspective. Seminar Press. Nueva York.

Binford, S. R. y Binford, L. R. 1968: New Perspectives in Archaeology. Aldine Publishing Co. Chicago.

Bintliff, J. y Pearce, M. (eds.) 2011: The death of anthropological theory?. Oxbow Books. Oxford.

Bird-David, N. 1999: "Animism" revisited: personhood, environment, and relational epistemology". Current Anthropology 40 (1): S67-91.

Blaser, M. 2014: "The political ontology of doing sameness ... and difference". Theorizing the Contemporary, Cultural Anthropology website, January 13, 2014. https://culanth.org/fieldsights/474-the-political-ontology-of-doing-difference-and-sameness

Bonte, P. 1974: "From ethnology to anthropology: on critical approaches in the human sciences". Critique of Anthropology 2: 36-57 y 3: 1-26.

Brown, B. 2001: "Thing theory". Critical Inquiry 28 (1): $1-22$.

Bryant L. 2011: The democracy of objects. Open Humanities. Ann Arbor.

Bryant, L.; Srnicek, N. y Harman, G. 2011: The speculative turn. Continental materialism and realism. Series Anamnesis re.press. Melbourne.

Clarke, D. L. 1968: Analytical Archaeology. Methuen and Co. Londres.

Clarke, D.L. 1972: Models in Archaeology. Methuen and Co. Londres.

Cochrane, E. E. y Gardner, A. (eds.) 2011: Evolutionary and interpretive archaeologies: a dialogue. Left Coast Press. Walnut Creek.

Comaroff, J. y Comaroff, J.L. (eds.) 2001: Milennial capitalism and the culture of neoliberalism. Duke University Press. Durham y Londres.
Conkey, M. W. 2003: "Has Feminism changed Archaeology?". Signs 28 (3): 867-880.

Conkey, M. W. y Spector, J. 1984: "Archaeology and the study of gender". Advances in Archaeological Method and Theory 7: 1-38.

Course, M. 2010: "Of words and fog: linguistic relativity and Amerindian ontology". Anthropological Theory 10 (3): 247-263.

Criado-Boado, F. 2016: "Tangled between paradigms in the neo-baroque era". Archaeological Dialogues 23 (2): 152-158.

Crutzen, P. y E. Stoermer 2000: “The Anthropocene". IGBP Newsletter 41: 17-18.

Davis, K. 2015: "The politics of the "turn". European Journal of Women's Studies 22 (2): 125-128.

DeLanda, M. 2006: A new philosophy of society: Assemblage Theory and Social Complexity. Continuum. Londres y Nueva York.

Deleuze, G. y Guattari, F. 1980: Mille plateaux. Éditions de Minuit. París.

Descola, P. 1992: "Societies of nature and the nature of society". En A. Kuper (ed.): Conceptualizing Society. Routledge. Londres: 107-126.

Descola, P. 1996: "Constructing natures: symbolic ecology and social practice". En P. Descola, P. y Pálsson, G. (eds.): Nature and society: anthropological perspectives. Routledge. Londres: 82-102

Descola, P. 2005: Par-delà nature et culture. Éditions Gallimard. París.

Descola, P. y Pálsson, G. (eds.) 1996: Nature and society: anthropological perspectives. Routledge. Londres.

Díaz-Andreu, M. 2007: A World History of nineteenth-century Archaeology: nationalism, colonialism and the past. Oxford University Press. Oxford.

Edgeworth, M. 2016: "Grounded objects: Archaeology and speculative realism". Archaeological Dialogues 23 (1): 93-113.

Fowler, C. 2013: The emergent past: a relational realist archaeology of Early Bronze Age mortuary practices. Oxford University Press. Oxford.

Fowler, C. y Harris, O. J. T. 2015: "Enduring relations: exploring a paradox of new materialism". Journal of Material Culture 20 (2): 127-48.

Fowles, S. 2016: "The perfect subject (postcolonial object studies)". Journal of Material Culture 21 (1): 9-27.

Gabriel, M. 2015: Fields of sense: a new realist Ontology. Edinburgh University Press. Edimburgo.

Gell, A. 1998: Art and agency: an anthropological theory. Oxford University Press. Oxford.

Gero, J. M.; Conkey, M. W. y Wright, M. 1991: Engendering Archaeology: women in Prehistory. Blackwell. Oxford.

Gilman, A. 1984: "Explaining the Upper Paleolithic revolution". En M. Spriggs (ed.): Marxist perspectives in Archaeology. Cambridge University Press. Cambridge: 115-126. 
González Abrisketa, O. y Carro-Ripalda, S. 2016: "La apertura ontológica de la antropología contemporánea". Revista de dialectología y tradiciones populares 71 (1): 101-128.

González-Ruibal, A. 2008: "Time to destroy: an Archaeology of supermodernity". Current Anthropology 49 (2): 247-279.

González-Ruibal, A. (ed.) 2013: Reclaiming Archaeology: beyond the tropes of modernity. Routledge. Londres.

González-Ruibal, A. 2014: "Returning to where we have never been: excavating the ruins of modernity". En B. Olsen y P. Pétursdóttir (eds.): Ruin memories: materialities, aesthetics and the Archaeology of the recent past. Routledge. Londres: 367-388.

Gosden, Ch. 1999: Anthropology and Archaeology: a changing relationship. Routledge. Londres.

Hamilakis, Y. 2013: Archaeology and the senses: human experience, memory, and affect. Cambridge University Press. Cambridge.

Hamilakis, Y. y Duke, P. 2007: Archaeology and capitalism: from ethics to politics. Left Coast Press. Walnut Creek.

Haraway, D. 1991: Simians, cyborgs and women: the reinvention of nature. Routledge. Nueva York.

Haraway, D. 2003: The companion species manifesto: dogs, people, and significant otherness. Prickly Paradigm Press. Chicago.

Haraway, D. J. 2007: When species meet. University of Minnesota Press. Minneapolis.

Harman, G. 2005: Guerrilla metaphysics. Open Court. Chicago.

Harman, G. 2009: Prince of networks: Bruno Latour and Metaphysics. Series Anamnesis re.press. Melbourne.

Harvey G. 2005: Animism: respecting the living world. Hurst \& Co. Londres.

Henare, A.; Holbraad, M. y Wastell, S. 2007: Thinking through things. Theorising artefacts ethnographically. Routledge. Londres.

Herschend, F. 2015: "Archaeology is History or it's History". En Ch. Hillerdal y J. Siapkas (eds.): Debating archaeological empiricism: the ambiguity of material evidence. Routledge. Nueva York: 125-143.

Hillerdal, Ch. y Siapkas, J. 2015 (eds.): Debating archaeological empiricism: the ambiguity of material evidence. Routledge. Nueva York.

Hodder, I. 1982a: Symbols in action. Ethnoarchaeological studies of material culture. Cambridge University Press. Cambridge.

Hodder, I. 1982b: The present past: an introduction to Anthropology for archaeologists. Batsford. Londres.

Hodder, I. 1982c: Symbolic and structural Archaeology. Cambridge University Press. Cambridge.

Hodder, I. 1986: Reading the past: current approaches to interpretation in Archaeology. Cambridge University Press. Cambridge.
Hodder, I. 2012: Entangled: an Archaeology of the relationships between humans and things. Wiley-Blackwell. Londres.

Hodder, I. 2016: Studies in human-thing entanglement. Creative Commons Attribution.

Hodder, I. y Hutson, S. 2003: Reading the past: current approaches to interpretation in Archaeology. Cambridge University Press. Cambridge.

Holbraad, M. 2012: Truth in motion: the recursive Anthropology of cuban divination. Chicago University Press. Chicago.

Holbraad, M. y Pedersen M. A. 2017: The ontological turn: an anthropological exposition. Cambridge University Press. Cambridge.

Ingold, T. 1994: "General introduction". En T. Ingold. Companion encyclopedia of Anthropology. Routledge. Londres: xiii-xxii.

Ingold, T. 2000: The perception of environment: essays on livelihood, dwelling and skill. Londres. Routledge.

Ingold, T. 2007: Lines: a brief history. Routledge. Londres.

Ingold, T. 2011: Being alive: essays on movement, knowledge and description. Routledge. Londres.

Ingold, T. 2012: "Toward an ecology of materials". Annual Reviews of Anthropology 41: 427-442.

Ingold, T. 2013: Making: Anthropology, Archaeology, Art and Architecture. Routledge. Londres.

Ingold, T. 2014: "That's enough about ethnography!". HAU, Journal of Ethnographic Theory 4 (1): 383-395.

Ingold, T. y Pálsson, G. 2013: Biosocial becomings. Integrating social and biological Anthropology. Cambridge University Press. Cambridge.

Jones, A. M. y Alberti, B. 2013: "Archaeology after interpretation". En B. Alberti, M. Jones y J. Pollard, J. (eds.): Archaeology after interpretation. Returning materials to archaeological theory. Left Coast Press. Walnut Creek: 15-35.

Kirksey, S. E. y Helmreich, S. 2010: "The emergence of multispecies ethnography". Cultural Anthropology 25 (4): 545-576.

Knauft, B. M. 2006: "Anthropology in the middle". Anthropological Theory 6 (4): 407-430.

Kohn, E. 2013: How forests think: toward an Anthropology beyond the human. University of California Press. Berkeley.

Kohn, E. 2015: "Anthropology of ontologies". Annual Review of Anthropology 44: 311-327.

Kristiansen, K. 2004: "Genes versus agents. A discussion of the widening theoretical gap in archaeology". Archaeological Dialogues 11 (2): 77-99.

Kristiansen, K.; Smejda, L. y Turek, J. 2015: Paradigm found: archaeological theory -present, past, future. Essays in Honour of Evzen Neustupny. Oxbow Books. Oxford.

Krotz, E. 1997: "Anthropologies of the South: their rise, their silencing, their characteristics". Critique of Anthropology 17 (3): 237-251. 
Krotz, E. 2015: "Las Antropologías segundas en América Latina: interpelaciones y recuperaciones". Cuadernos de Antropología Social 42: 5-17.

Laidlaw, J. 2012: “Ontologically challenged". Anthropology of this century 4. http://aotcpress.com/articles/ontologically-challenged/

Laidlaw, J. y Heywood, P. 2013: "One more turn and you're there". Anthropology of this century 7. http:// aotcpress.com/articles/turn/

Latour, B. y Woolgar, S. 1979: Laboratory life: the social construction of scientific facts. Sage. Londres.

Latour, B. 1987: Science in action. Harvard University Press. Cambridge.

Latour, B. 1988: The pasteurization of France. Harvard University Press. Cambridge.

Latour, B. 1991: Nous n'avons jamais été modernes: Essai d'anthropologie simétrique. La Découverte. París.

Latour, B. 1996: Aramis, or the Love of Technology. Harvard University Press. Cambridge.

Latour, B. 1999a: Politiques de la nature. Comment faire entrer les sciences en démocratie. La Découverte. París.

Latour, B. 1999b: Pandora's hope: essays on the reality of science studies. Harvard University Press. Cambridge.

Latour, B. 2005: Reassembling the social: an introduction to Actor-Network Theory. Oxford University Press. Oxford.

Latour, B. 2012: Enquête sur les modes d'existence. Une anthropologie des modernes. La Découverte. París.

Latour, B. 2013a: An inquiry into modes of existence. An Anthropology of the moderns. Harvard University Press. Cambridge.

Latour, B. 2013b: "Another way to compose the common world". 132 AAA Annual Meetings, Chicago, IL, November 23. http://www.bruno-latour. $\mathrm{fr} /$ sites/default/files/132-AAA-CHICAGO-PHIlANTH-2013.pdf

Lévi-Strauss, C. 1960: "Le trois sources de la réflexion ethnologique". Revue de l'enseignement supérieur 1: 43-50.

Lucas, G. 2012: Understanding the archaeological record. Cambridge University Press. Cambridge.

Lucas, G. 2016: "The paradigm concept in archaeology". World Archaeology. doi: 10.1080/ 00438243.2016 .1252688$.

Marshall, Y. y Alberti, B. 2014: "A matter of difference: Karen Barad, Ontology and archaeological bodies". Cambridge Archaeological Journal 24 (1): 19-36.

Martin, A.M. 2013: Archaeology beyond Postmodernity. A science of the social. Altamira Press. Plymouth.

Mateo Dieste, J. L. y Coello de la Rosa, A. 2016: Elogio de la Antropología histórica: enfoques, métodos y aplicaciones al estudio del poder y del colonialismo. Prensas Universitarias de Zaragoza. Zaragoza.
McGuire, R. 1992: A marxist Archaeology. Percheron Press. Nueva York.

McGuire, R. 2009: Archaeology as political action. University of California Press. Berkeley.

McGuire, R. y Paynter, R. 1991: The Archaeology of inequality. Oxford. Blackwell.

Miller D. 1987: Material culture and mass consumption. Blackwell. Oxford.

Miller, D. 1998: "Why some things matter". En D. Miller (ed.): Material cultures: why some things matter. UCL Press. Londres: 3-21.

Miller D. 2005: "Materiality: an introduction". En D. Miller (ed.): Materiality. Duke University Press. Durham NC: $1-50$.

Nichols, D. L.; Joyce, R. A. y Gillespie, S.. 2003: "Is Archaeology Anthropology?". En S. D. Gillespie y D.L. Nichols (eds.): Archaeology is Anthropology. American Anthropological Association. Arlington: 3-16.

Olsen, B. 2003: "Material culture after text: Re-membering things". Norwegian Archaeological Review 36 (3): 87-104.

Olsen, B. 2010: In defense of things: Archaeology and the ontology of objects. Altamira Press. Plymouth.

Olsen, B. 2012a: "After interpretation: remembering Archaeology". Current Swedish Archaeology 20: 11-34.

Olsen, B. 2012b: "Symmetrical Archaeology". En I. Hodder (ed.): Archaeological Theory Today. Polity Press. Cambridge: 208-228.

Olsen, B.; Shanks, M.; Webmoor, T. y Witmore, C. 2012: Archaeology: the discipline of things. University of California Press. Berkeley.

Olsen, B. y Witmore, C. 2015: "Archaeology, symmetry and the ontology of things. A response to critics". Archaeological dialogues 22 (2): 187-197.

Paynter, R. 1988: "Steps to an archaeology of capitalism”. En M. Leone y P. Potter (eds.): The recovery of meaning. Smithsonian Institution Press. Washington: 407-33.

Pedersen, M.A. 2012: “Common Nonsense: A review of certain recent reviews of the Ontological Turn". Anthropology of this century 5. http://aotcpress.com/ articles/common_nonsense/

Phillips, Ph. 1955: "American Archaeology and General Anthropological Theory”. Southwestern Journal of Anthropology 11 (3): 246-250.

Preucel, R.W. 2006: Archaeological Semiotics. Blackwell. Malden.

Preucel, R.W. 2016: "Pragmatic Archaeology and semiotic mediation". Semiotic Review 4: Im/materialities, November 2016 https://semioticreview.com/ ojs/index.php/sr/article/view/11

Preucel, R. W. y Mrozowski, S. A. (eds.) 2010: Contemporary Archaeology in theory: the new pragmatism. Blackwell. Londres.

Ramos, A. R. 2012: "The politics of perspectivism". Annual Review of Anthropology 41: 481-494. 
Restrepo, E. y Escobar, A. 2005: "'Other anthropologies and anthropology otherwise': steps to a world anthropologies framework". Critique of Anthropology 25 (2): 99-129.

Ribeiro, G. L. y Escobar, A. 2008: Antropologías del mundo: transformaciones disciplinarias dentro de sistemas de poder. Envión Editores-Ciesas-Wenner Gren. Popayán.

Ribeiro, A. 2016: "Archaeology will be just fine". Archaeological Dialogues 23 (2): 146-151.

Shanks, M. 1992: Experiencing the past: on the character of archaeology. Routledge. Londres.

Shanks, M. 2007: "Symmetrical Archaeology". World Archaeology 39 (4): 589-596.

Shanks, M. y Tilley, C. 1987a: Re-constructing Archaeology. Theory and practice. Cambridge University Press. Cambridge.

Shanks, M. y Tilley, C. 1987b: Social theory and Archaeology. Polity Press. Cambridge.

Shennan, S. 2004: "Analytical archaeology". En J. Bintliff (ed.): A companion to Archaeology. Blackwell. Oxford: 3-20.

Simondon, G. 1964: L'individu et sa génèse physico-biologique. PUF. París.

Spector, J. M. 1991: "What this awl means: towards a feminist archaeology". En J. M. Gero, M. W. Conkey y M. Wright (eds.): Engendering archaeology: women and prehistory. Blackwell. Oxford: 388-406.

Spriggs, M. (ed.) 1984: Marxist perspectives in Archaeology. Cambridge University Press. Cambridge.

Strathern, M. 1988: The gender of the gift. Problems with women and problems with society in Melanesia. University of California Press. Berkeley.

Strathern, M. 1991: Partial connections. Rowman and Littlefield. Savage.

Strathern, M. 1995: Schifting contexts: Transformations in Anthropological knowledge. Routledge. Londres.

Thiéry, O. y Houdart, S. 2011: Humains, non-humains. Comment repeupler les Sciences Sociales. La Découverte. París.

Thomas, J. 2015: "The future of archaeological theory". Antiquity 89 (348): 1287-1296.

Tilley, C. 1994: A phenomenology of landscape. Places, paths, and monuments. Berg. Londres.

Tilley, C. 1999: Metaphor and material culture. Blackwell. Oxford.

Trigger, B.G. 1989: A history of archaeological thought. Cambridge University Press. Cambridge.

Trigger, B. 1993: "Marxism in contemporary Western archaeology". En M. B. Schiffer (ed.): Archaeological Method and Theory. University of Arizona Press. Tucson: 159-200.

Trigger, B. 1995: "Archaeology and the integrated circus". Critique of Anthropology 15 (4): 319-335.

Vasileva, B. 2015: "Stuck with/in a "turn": Can we metaphorize better in Science and Technology Studies?". Social Studies of Science 45 (3): 454-461.
Vigh, H. E. y Sausdal, D. B. 2014: "From essence back to existence: anthropology beyond the ontological turn". Anthropological Theory 14 (1): 49-73.

Viveiros de Castro, E. 1998: "Cosmological deixis and Amerindian perspectivism". The Journal of the Royal Anthropological Institute 4 (3): 469-88.

Viveiros de Castro, E. 2003: "(anthropology) AND (science)". After-dinner speech at "Anthropology and Science', the $5^{\text {th }}$ Decennial Conference of the Association of Social Anthropologists of Great Britain and Commonwealth, 14 ${ }^{\text {th }}$ July 2003. Manchester Papers in Social Anthropology 7. University of Manchester. Manchester.

Viveiros de Castro, E. 2009: Métaphysiques cannibales. PUF. París.

Viveiros de Castro, E. 2011: "Zeno and the art of Anthropology: of lies, beliefs, paradoxes and other truths". Common Knowledge 17 (1): 128-145.

Wagner, R. 1978: Lethal speech: Daribi myth as symbolic obviation. Cornell University Press. Ithaca.

Wagner, R. 1981: The Invention of Culture. University of Chicago Press. Chicago.

Webmoor, T. y Witmore, C.L. 2008: "Things are us! A commentary on human/things relations under the banner of a 'social' archaeology". Norwegian Archaeology Review 41 (1): 1-18.

Wiley, A. 1991: "Gender theory and the archaeological record. Why is there no archaeology of gender?". En J. M. Gero, M. W. Conkey y M. Wright (eds.): Engendering Archaeology: women in Prehistory. Blackwell. Oxford: 31-54.

Willerslev R. 2007: Soul hunters: hunting, animism, and personhood among the siberian Yukaghirs. University of California Press. Berkeley.

Willerslev, R. 2012: "Laughing at the spirits in North Siberia: is animism being taken too seriously?". E-Flux Journal 36 (7): 13-22.

Willey, G. R. y Phillips, Ph. 1958: Method and Theory in American Archaeology. University of Chicago Press. Chicago.

Witmore, C. L. 2007: "Symmetrical archaeology: excerpts from a Manifesto". World Archaeology 39 (4): 546-562.

Witmore, C. L. 2014: "Archaeology and the new materialisms". Journal of Contemporary Archaeology 1 (2): 203-246.

Witmore, C. 2015: "Archaeology and the second empiricism". En Hillerdal, C. y J. Siapkas (eds.) Debating Archaeological Empiricism. The Ambiguity of Material Evidence. Routledge. London: 37-67.

Woolgar, S. y Lezaun, J. 2013: "The wrong bin bag: a turn to ontology in science and technology studies?". Social Studies of Science 43 (3): 321-340.

Woolgar, S. y Lezaun, J. 2015: "Missing the (question) mark? What is a turn to ontology?". Social Studies of Science 45 (3): 462-467. 